\title{
Temporal patterns of rat behaviour in the central platform of the elevated plus maze. Comparative analysis between male subjects of strains with different basal levels of emotionality
}

\author{
M. Casarrubea ${ }^{a}, *$, F. Faulisi $^{\mathrm{a}}$, F. Caternicchia ${ }^{\mathrm{a}}$, A. Santangelo ${ }^{\mathrm{b}}$, G. Di Giovanni $^{\mathrm{c}, \mathrm{d}}$, \\ A. Benigno ${ }^{a}$, M.S. Magnusson ${ }^{\mathrm{e}}$, G. Crescimanno $^{\mathrm{a}}$ \\ a Dept of Experimental Biomedicine and Clinical Neurosciences, Human Physiology Section "Giuseppe Pagano", Laboratory of Behavioral Physiology, \\ University of Palermo, Palermo, Italy \\ ${ }^{\mathrm{b}}$ Dept of Neuroscience, Psychology, Drug Research and Child Health, University of Florence, Florence, Italy \\ ${ }^{\mathrm{c}}$ Dept of Physiology and Biochemistry, Faculty of Medicine and Surgery, University of Malta, Msida, Malta \\ d School of Biosciences, Cardiff University, Cardiff, UK \\ e Human Behavior Laboratory, University of Iceland, Reykjavik, Iceland
}

\section{H I G H L I G H T S}

- The central platform is the zone of the EPM where the rat selects the arm to explore.

- The behaviour of two strains of rats has been studied in the central platform.

- The temporal structure of rat behaviour has been analyzed through T-pattern analysis.

- Results indicate two different approaches in the interaction with the environment.

\section{G R A P H I C A L A B S T R A C T}

Wistar and DA/Han rats are characterized by a different basal level of emotionality. The application of multivariate T-pattern analysis to study their behaviour in the central platform revealed two completely different behavioural strategies. The illustration shows the ethogram and, on the right, all the different temporal patterns detected in the two strains.

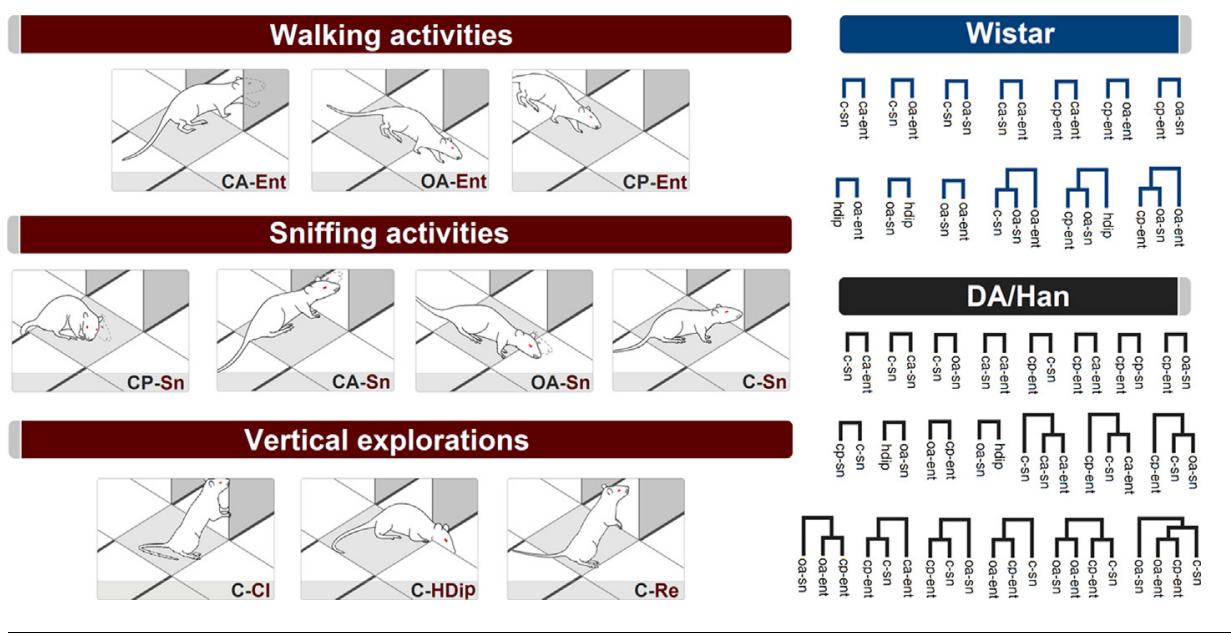

\section{A B S T R A C T}

We have analyzed the temporal patterns of behaviour of male rats of the Wistar and DA/Han strains on the central platform of the elevated plus maze. The ethogram encompassed 10 behavioural elements. Durations, frequencies and latencies showed quantitative differences as to walking and sniffing activities. Wistar rats displayed significantly lower latency and significantly higher durations and frequencies of walking activities. DA/Han rats showed a significant increase of sniffing duration. In addition, DA/Han rats showed a significantly higher amount of time spent in the central platform. Multivariate T-pattern

\footnotetext{
* Corresponding author at: Bio.Ne.C., Human Physiology Section “Giuseppe Pagano”, Laboratory of Behavioral Physiology, University of Palermo, Corso Tukory n 129 , 90134 Palermo, Italy. Tel.: +39 0916555848.

E-mail addresses: maurizio.casarrubea@unipa.it (M. Casarrubea), fabiana.faulisi@unipa.it (F. Faulisi), filippocaternicchia@virgilio.it (F. Caternicchia), a.santangelo@ymail.com (A. Santangelo), giuseppe.digiovanni@um.edu.mt (G. Di Giovanni), arcangelo.benigno@unipa.it (A. Benigno), msm@hi.is (M.S. Magnusson)، giuseppe.crescimanno@unipa.it (G. Crescimanno). 
Keywords:

Anxiety

Elevated plus maze

Central platform

Wistar

DA/Han

T-pattern analysis

Male rat analysis revealed differences in the temporal organization of behaviour of the two rat strains. DA/Han rats showed (a) higher behavioural complexity and variability and (b) a significantly higher mean number of T-patterns than Wistar rats.

Taken together, T-pattern analysis of behaviour in the centre of the elevated plus maze can noticeably improve the detection of subtle features of anxiety related behaviour. We suggest that T-pattern analysis could be used as sensitive tool to test the action of anxiolytic and anxiogenic manipulations.

(c) 2015 Elsevier B.V. All rights reserved.

\section{Introduction}

With thousands of published papers so far, the elevated plus maze (EPM) is, probably, the most popular and used experimental apparatus to assess anxiety-related behaviour in rodents (Web of Knowledge, 2015). The EPM usefulness has spread towards the understanding of the basis of emotionality related to learning and memory, addiction and withdrawal, as well as phobia, generalized anxiety and post-traumatic stress (Carobrez and Bertoglio, 2005). The apparatus consists of an elevated plus-shaped platform comprehending four arms extended from a common central platform: two facing arms lack of enclosures and are more brightly lit (so called open arms), the remaining two facing arms are surrounded by opaque enclosures and, therefore, are less illuminated (so called closed arms). Basically, the rationale of the utilization of EPMs orbits around the evidence that naïve rodents, usually very cautious in novel and unfamiliar environments, show a clear-cut preference for the closed arms and only subjects with a reduced anxiety level increase their activity in the open arms (Carobrez and Bertoglio, 2005). In such a context, the central platform of the apparatus is the zone of the EPM where the animal selects "what to do" or, in other terms, the zone where decision making processes are carried out (Rodgers et al., 1992; Rodgers and Johnson, 1995; Cruz et al., 1994; Casarrubea et al., 2015a). Simply stated, the central platform of the EPM, on the basis of its position and structural characteristics, is crucial in moulding the actual behaviour of the rodent in the apparatus. Consistently, the importance of studies evaluating the behaviour of the rodent in the central platform has been underlined since two decades ago (Rodgers and Dalvi, 1997). Even so, surprisingly scanty data do take into consideration the behaviour of the rodent in the central platform of the maze. To shed light on this matter, we have recently focused on the behaviour of two strains of rats with different emotional reactivity, analyzed in the central platform of the EPM (Casarrubea et al., 2015a). By using a multivariate approach based on transition matrices elaboration we have demonstrated that rats with different reactivity to anxiogenic stimuli present profound differences in behavioural switching, that is, the ability to move from a behaviour to another one. However, beyond transitions among the elements of the behavioural repertoire, an interesting issue still represents matter of debate deserving supplementary experimental analyses: the real time structure of rat behaviour in the central platform. Actually, transition matrices and related elaborations (e.g. dendrograms, stochastic path diagrams or adjusted residuals) (Spruijt and Gispen, 1984; Espejo and Mir, 1993; Espejo, 1997; Lino-de-Oliveira et al., 2005; Casarrubea et al., 2009, 2010a, 2010b, 2011, 2012; Kalueff and Tuohimaa, 2004; Van Den Berg et al., 1999; Vanderschuren et al., 1996) do represent the behaviour resembling a snapshot of the entire period of observation: information is not provided on the real time structure of behaviour. On the contrary, by means of T-pattern analysis, the real time structure of behaviour can be minutely analyzed (Magnusson, 2000; Casarrubea et al., 2009, 2013a, 2013b, 2014, 2015b). In present paper, we use multivariate T-pattern analysis to investigate the temporal structure of behaviour in the central platform of the maze in Wistar and in DA/Han strain, the latter has been demonstrated to possess a behavioural profile compatible with a high anxiety level (King, 1999; Mechan et al., 2002; Chapillon et al., 2002; Roy and Chapillon, 2002, 2004; Roy et al., 2003, 2009; Casarrubea et al., 2013b, 2015a). By providing information on the temporal structure of the behaviour in the central platform, new insights on rat behavioural dynamics in the elevated plus maze can be provided. A particular attention will be dedicated to methodological aspects of the experiment.

\section{Method}

\subsection{Subjects}

Ten male Wistar and ten male DA/Han, SPF, rats were used. Both Wistar and DA/Han subjects were three months old. Animals were born in the animal facility of the University of Rouen (France) and breeders originated from Janvier (Le Genest-St-Isle, France). Rats were housed in groups of three in a room maintained at the constant temperature of $21 \pm 2{ }^{\circ} \mathrm{C}$, under the following light/dark cycle: light on = 12 noon; light off = 12 midnight. Food and water were available "ad libitum".

\subsubsection{Ethical statement}

All efforts were carried out to minimize the number of animals used and their suffering. The experiments were conducted in accordance with the European Communities Council Directive 86/609/EEC concerning the protection of animals used for experimental scientific purposes.

\subsection{Experimental apparatus}

The experimental apparatus was made of Perspex. Each arm was $50 \mathrm{~cm}$ long and $10 \mathrm{~cm}$ wide. The apparatus was elevated at a height of $50 \mathrm{~cm}$ above the floor. The closed arms were surrounded by a $50 \mathrm{~cm}$ opaque wall, the open ones presented $0.5 \mathrm{~cm}$ edges to maximize open-arm entries (Treit et al., 1993). The floor of the apparatus was covered with grey plastic. Environmental temperature in testing room was continuously measured and maintained equal to the temperature in housing room. The testing room was illuminated with a dim white light providing 100 lux in the open arms, 85 lux in the central platform and 50 lux in the closed arms.

\subsection{Experimental procedure}

Rats were transported from housing to testing room inside their home-cages to minimize transfer effect. Each rat was allowed to acclimate for $30 \mathrm{~min}$ far from the observational apparatus to avoid possible visual and/or olfactory influences. Each subject, experimentally naïve, was placed in the central platform of the maze, facing an open arm, and allowed to freely explore for $5 \mathrm{~min}$. After each observation, the EPM was cleaned with ethyl alcohol (10\%) to remove scent cues left from the preceding subject. Rat behaviour 


\section{Central Platform of the EPM}

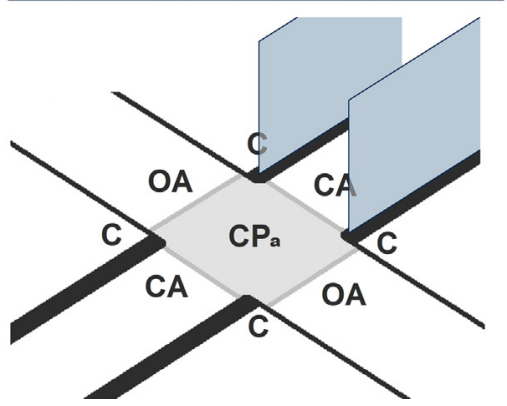

Fig. 1. Central platform of the elevated plus maze. Only the walls of one closed arm have been represented. $\mathrm{CPa}=$ Central Platform Area; $\mathrm{CA}=$ Closed Arm Zones; $\mathrm{OA}=$ Open Arm Zones; $\mathrm{C}=$ Corner Zones (closed-open arm junction and external $90^{\circ}$ angle comprised between the two arms).

was recorded by means of a video camera, and video files were stored in a PC and subsequently analyzed.

\subsection{Central platform and ethogram}

In the EPM for rats, the central platform (CP) is a $10 \mathrm{~cm} \times 10 \mathrm{~cm}$ square in the centre of the apparatus (Fig. 1). On the basis of the adjacent arms and enclosures, the $\mathrm{CP}$ presents nine subregions: $\mathrm{a}$ central area $(\mathrm{CPa})$, two borders between the $\mathrm{CPa}$ and the open arms (OA), two borders between the CPa and the closed arms (CA), and finally, four corners (C). By taking into consideration these regions and borders (Fig. 1), it has been possible to arrange an ethogram encompassing 10 behavioural components organized in three main categories (Fig. 2): Walking activities, namely, behavioural components where the animal walks from an adjacent arm to the CPa or vice versa (head and front-paws across the border line between the two regions: CA-Ent, OA-Ent, CP-Ent, see Fig. 2); Sniffing activities, namely, behavioural components characterized by the sniffing of the $\mathrm{CPa}$ and adjacent zones (all four paws in $\mathrm{CPa}$, rapid scanning head movements often associated with movement of vibrissae: $\mathrm{CP}-$ Sn, CA-Sn, OA-Sn, C-Sn, see Fig. 2); and, finally, Vertical explorations, that is, behavioural components where the animal, maintaining a fixed position on the $\mathrm{CPa}$, explores above or below the ground $(\mathrm{C}-\mathrm{Cl}$, C-HDip, C-Re, see Fig. 2).

\subsection{Data analysis}

Video files were frame-by-frame analyzed using a personal computer equipped with a software coder (The Observer, Noldus IT - The Netherlands). Event log files, obtained from the software coder, were analyzed by means of quantitative approaches and by means of T-pattern analysis (Theme software, Noldus IT - The Netherlands; Patternvision Ltd - Iceland).

\subsubsection{Quantitative analyses}

To evaluate the amount of time each subject spent in the $\mathrm{CP}$, in the $\mathrm{OA}$ and in the CA, mean durations have been measured. To evaluate the number of entries in the $\mathrm{CP}$, in the $\mathrm{OA}$ and in the $\mathrm{CA}$ mean occurrences have been calculated. To calculate the amount of time each subject spent in walking activities, sniffing activities and vertical explorations, mean durations have been measured. In addition, to assess the number of behavioural components performed by each subject, mean occurrences of all the behavioural components have been measured as well. Finally, to evaluate the onset of the behavioural categories, mean latencies of the first occurrence of walking activities, sniffing activities and vertical explorations have been calculated. All the quantitative analyses have been carried on the basis of the event log files obtained from coding process.

\subsubsection{Multivariate T-pattern analysis}

To study the temporal architecture of rat behaviour in the $\mathrm{CP}$, multivariate T-pattern analysis has been applied. Basically, Tpatterns are sequences of events in time such that the order of the
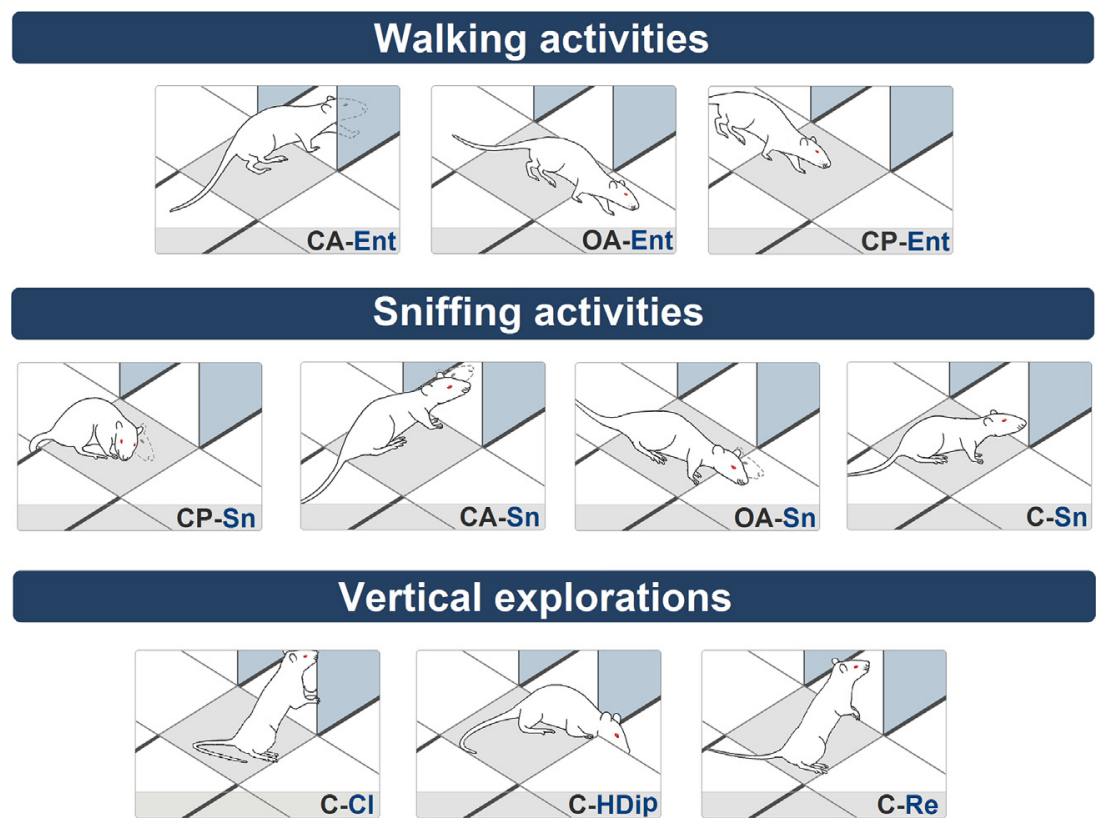

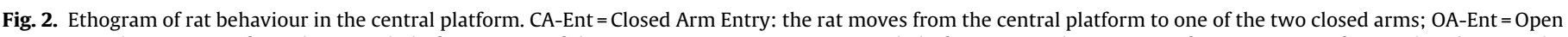

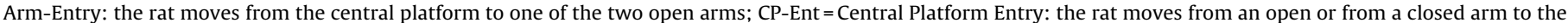

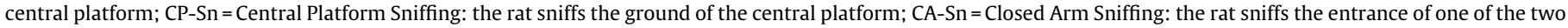

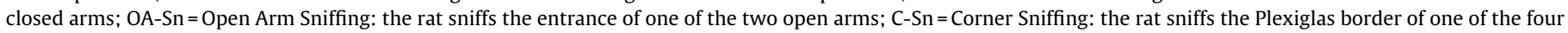

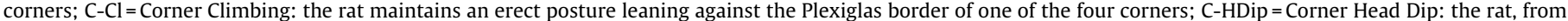

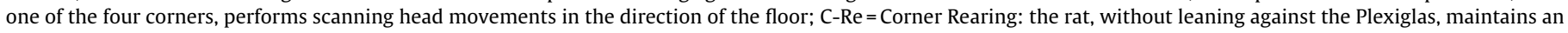
erect posture facing one of the four corners. 


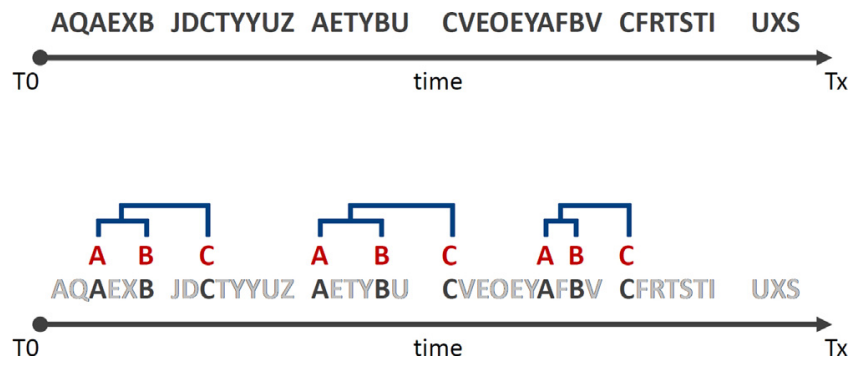

Fig. 3. Example of T-pattern. Albeit a repeated sequence of three events is present, the detection of such sequences is not an easy task if only upper row is observed. On the contrary, if the "background noise" is removed the $((\mathrm{AB}) \mathrm{C})$ pattern becomes evident even at a very first glance.

events is the same each time and the distances between them are significantly fixed as defined by a statistically defined critical interval relationship. Given three hypothetical behavioural elements "A", "B" and "C", occurring during a time period T0-Tx (Fig. 3), as a first step, an algorithm compares the distributions of each pair of the elements $A$ and $B$ searching for a time window so that, more often than chance expectation, $\mathrm{A}$ is followed by $\mathrm{B}$ within that time window. In this case, $A$ and $B$ are by definition a T-pattern indicated as (A B). In a second step, such first level T-patterns are marked and considered as potential A or B terms in higher patterns, for example, ((A B) C). Thus, more complex patterns may be created following this hierarchical bottom-up detection process up to any level. When no more patterns are found, the search stops. A more detailed description of theories and concepts behind Tpattern analysis and related detection procedures can be found in our recent comprehensive review (Casarrubea et al., 2015b).

\subsubsection{Statistics}

Concerning mean latencies, mean durations and mean frequencies, to compare possible significant differences of values detected in Wistar and in Da/Han rats, Student's t-test has been employed. As to T-pattern analysis, each critical interval underlines the detection of a statistically significant constraint. Notwithstanding, the extremely high number of possibilities of such relationships in data with hundreds or even thousands occurrences of behavioural events might raise the question whether the observed T-patterns are detected only by chance. Theme deals with such an issue by repeatedly randomizing and re-analysing the original data, using exactly the same search parameters used with the real behavioural data. The average number of patterns of each length detected in the randomized data is then compared with that obtained from the original data.

\subsection{Graphical representations}

Mean durations, mean frequencies and mean latencies have been represented with histograms. All T-patterns detected have been illustrated by means of terminal strings (that is, the text list of the events in sequence for each pattern) and tree structures. In addition, the onset of all patterns has been represented by means of a rasterplot. Finally, T-patterns length distribution in real and in random generated data have been represented by means of histograms.

\section{Results}

\subsection{Quantitative analyses}

\subsubsection{EPM zones}

Mean time $\pm \mathrm{SE}(\mathrm{s})$ each subject spent in the $\mathrm{CP}$, in the $\mathrm{OA}$ and in the CA is illustrated in Fig. 4A. Student's t-test revealed significant
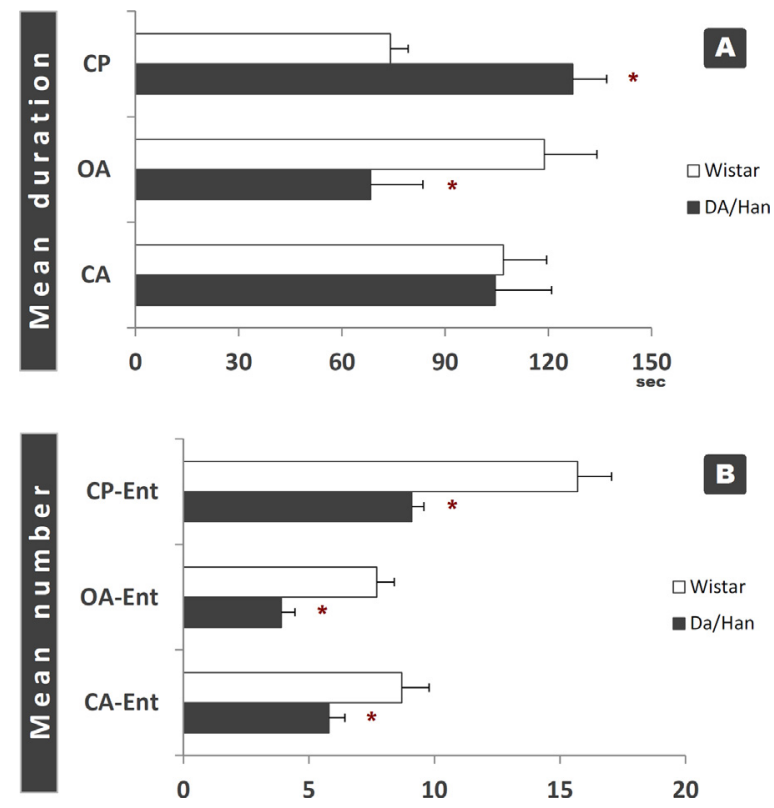

Fig. 4. Panel $A$ : mean duration $\pm S E(s)$ of permanence in central platform $(C P)$, open arms (OA) and closed arms (CA) in Wistar and DA/Han rats. X-Axis = seconds. Y-Axis: zones of the maze. Panel B: mean number of entries in central platform (CP-ent), open arms (OA-ent) and closed arms (CA-ent). X-axis = number of entries: $\mathrm{Y}$-axis $=$ zones of the maze where the rat enters. ${ }^{*} p<0.05$ (Student's $t$ test). Data obtained from the analysis of 10 Wistar and 10 DA/Han rats.

differences between the two strains for the time spent in $\mathrm{CP}$ $(p<0.0001)$ and for the time spent in OA $(p<0.05)$; no significant difference has been detected for the time spent in the CA $(p=0.908)$.

Mean number of entries in the $\mathrm{CP}$, in the $\mathrm{OA}$ and in the CA is illustrated in Fig. 4B. Student's t-test revealed significant differences between the two strains for the number of entries in $\mathrm{CP}(p<0.0001)$, in $\mathrm{OA}(p<0.0001)$ and in $\mathrm{CA}(p<0.05)$.
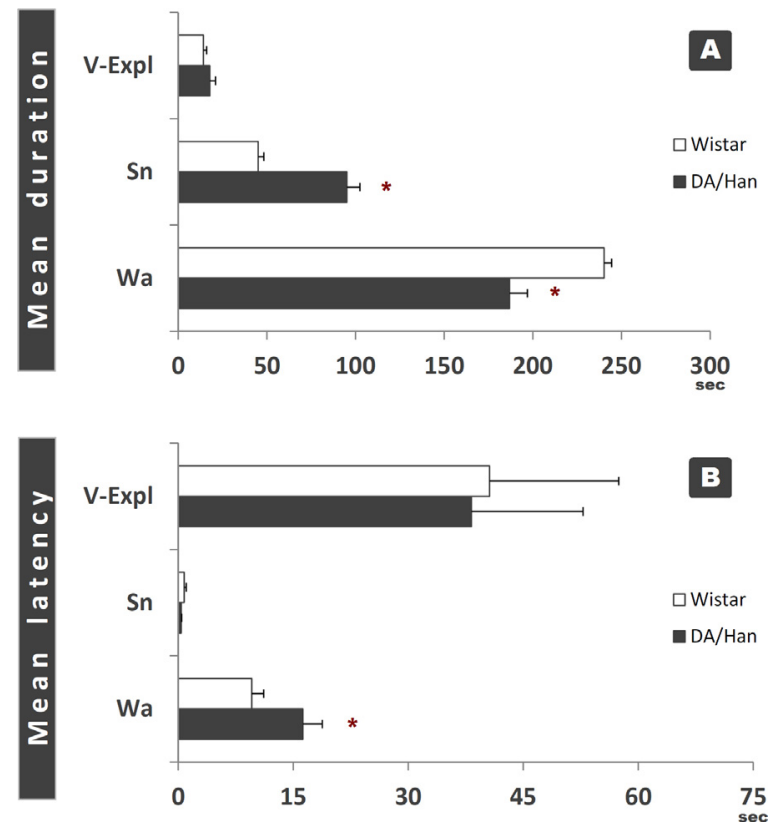

Fig. 5. Panel A: mean duration $\pm S E$ (s) of the walking activities (Wa), sniffing activities (Sn) and vertical explorations (V-Expl). X-axis = mean duration (s); Y-axis = behavioural categories. Panel B: mean latencies \pm SEM (s) of the first occurrences of walking activities (Wa), sniffing activities ( $\mathrm{Sn}$ ) and vertical explorations (V-Expl). X-axis = mean latency (s); Y-axis = behavioural categories. ${ }^{*} p<0.05$ (Student's $t$ test). Data obtained from the analysis of 10 Wistar and $10 \mathrm{DA} / \mathrm{Han}$ rats. 
Wistar

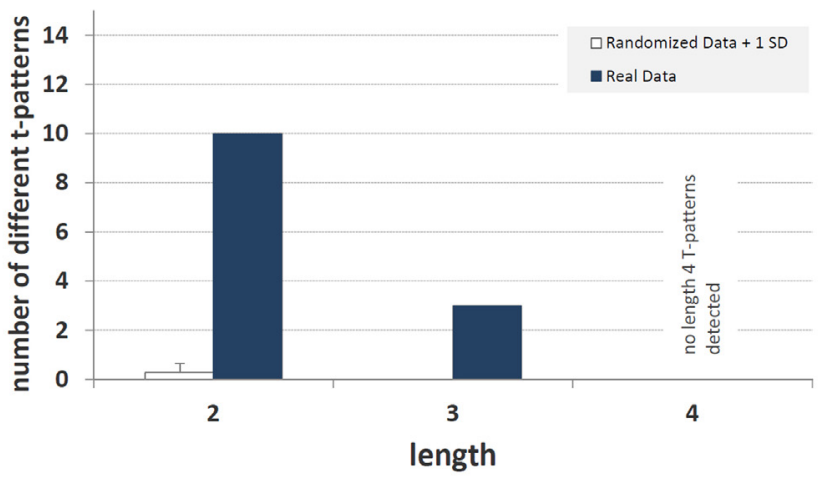

DA/Han

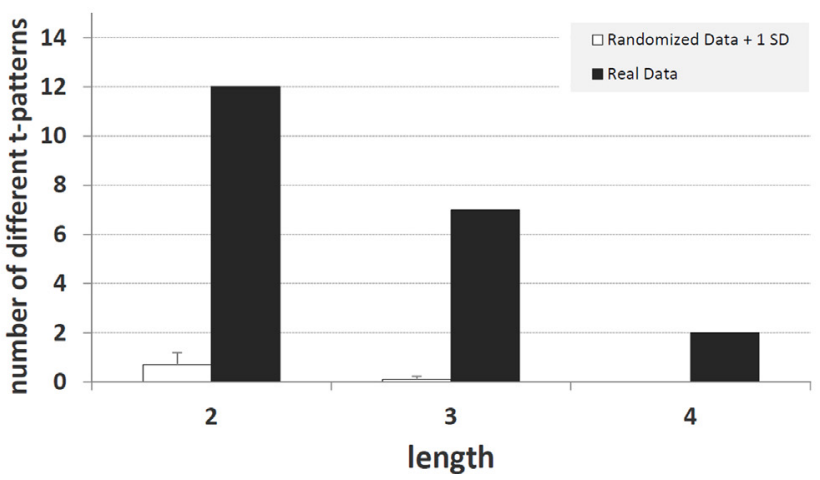

Fig. 6. Number of different T-patterns on the basis of their length. $X$-axis = number of events encompassed in the T-pattern structure. Y-axis = number of T-patterns of different composition. Dark bars = real data; white bars = average \pm 1 SD after 10 random runs. Data obtained from the analysis of 10 Wistar and $10 \mathrm{DA} / \mathrm{Han}$ rats.

\subsubsection{Performed activities}

Mean time $\pm S E(s)$ each subject spent in walking activities, sniffing activities and vertical explorations is illustrated in Fig. 5A. Student's t-test revealed significant differences between the two strains for walking $(p<0.0001)$ and for sniffing activities $(p<0.0001)$; no significant difference has been detected for vertical explorations $(p=0.350)$.

Mean latency $\pm S E(s)$ of the first occurrence of walking activities, sniffing activities and vertical explorations is illustrated in Fig. 5B. Student's $t$-test revealed significant differences between the two strains for walking activities ( $p<0.05$ ); no significant differences have been detected for sniffing activities $(p=0.179)$ and for vertical explorations $(p=0.917)$.

Finally, concerning mean number of elements, regardless the type of activity, Wistar rats performed, during the 5 min observation, an average of $69.40 \pm 5.02$ behavioural elements, DA/Han rats $66.2 \pm 3.78$. No significant differences between the two values have been detected $(p=0.617)$.

\subsection{T-pattern analysis}

Wistar rats performed 13 different T-patterns distributed and composed as follows: 10 encompassing two events and 3 with three events in their structure. No higher order patterns have been detected (Figs. 6 and 7). DA/Han rats presented 21 different patterns: 12 encompassing two events, 7 with three events and 2 with four events (Figs. 6 and 7). Overall, 554 T-patterns occurred in Wistar and 792 in DA/Han rats (Fig. 7, "Occs" column). Events in sequence, respective occurrences and tree structures are illustrated in Fig. 7 both for Wistar and DA/Han rats. Fig. 8 illustrates the first occurrence of all the T-patterns detected. Rasterplots in Fig. 9 present, for both strains, the onset of all the T-pattern detected during the $5 \mathrm{~min}$ of observation. Finally, evaluation of the mean number of T-patterns in each group revealed a significantly $(p<0.0001)$ higher number of occurrences in DA/Han subjects (Fig. 10). In both strains, comparison of real and random generated data showed a negligible amount of T-patterns in random data (Fig. 6).

\section{Discussion}

The present study provides new insights on the behaviour of two strains of rats, namely Wistar and DA/Han, characterized by different reactivity to the anxiogenic stimuli, and analyzed in the EPM's central platform.

The DA/Han rat (often referred as Dark Agouti) is known for its peculiar emotional reactivity in comparison with other strains (King, 1999; Mechan et al., 2002; Roy et al., 2009). In brief, this strain has been described as exhibiting high anxiety levels in studies utilizing various experimental devices such as a modified plus maze, standard plus maze, open field or automatic activity meter (King, 1999; Mechan et al., 2002). In our laboratory the DA/Han rat, on the basis of observations with the elevated plus maze, has been demonstrated to possess a behavioural profile compatible with a higher anxiety level in comparison with Wistar rats (Casarrubea et al., 2013b, 2015a). In detail, we have demonstrated strong differences between Wistar and DA/Han rats in terms of durations and percent distributions of specific behavioural components in the CP (e.g. a clear prevalence of sniffing activities) (Casarrubea et al., 2015a). In addition, by using a multivariate approach based on transition matrices we have revealed a different behavioural organization of the two strains.

\subsection{Transition matrices: advantages and drawbacks}

Transition matrices can be graphically expressed. On this subject, dendrograms or path diagrams (Casarrubea et al., 2009, 2010b, 2012, 2015a) are useful representations since they allow an immediate understanding of relationships between different groups of behavioural elements. Hence, the results provided by this kind of approach could represent a great advantage in terms of synopsis and straightforwardness. However, it is also important to mention that a transition matrix and all the related elaborations such as, for instance, Markovian analyses, adjusted residuals or, as abovementioned, cluster analyses (Casarrubea et al., 2015a), describe the comprehensive period of observation, without considering the temporal dimension and possible temporal relationships among the components of the behavioural repertoire. In other terms, transition matrices "summarize" the behaviour of the subject like a "snapshot" of the comprehensive period of observation: no information concerning time are provided. In the present article, by using multivariate T-pattern analysis, we fill such a gap on the behaviour of the rat in the central platform of the elevated plus maze. For the first time, indeed, information on the fine temporal structure of behaviour in this zone of the maze is provided.

\subsection{T-patterns}

The great advantage of multivariate T-pattern analysis lies in its ability to provide information concerning the relationships among behavioural events occurring during the observation.

On this subject, present results highlight that both Wistar and DA/Han rats present, in the central platform, a complex behaviour organized on the basis of several sequences of events with highly significant constraints on the interval length separating them. 


$\begin{array}{cccc} & & & \text { Wistar } \\ \text { TP\# } & \text { Terminal string } & \text { Occs } & \text { Length } \\ 1 & \text { (c-sn ca-ent ) } & 82 & 2 \\ 2 & \text { (c-sn oa-ent) } & 36 & 2 \\ 3 & \text { (c-sn oa-sn ) } & 48 & 2 \\ 4 & \text { (ca-sn ca-ent) } & 32 & 2 \\ 5 & \text { (cp-ent ca-ent) } & 84 & 2 \\ 6 & \text { (cp-ent oa-ent) } & 66 & 2 \\ 7 & \text { (cp-ent oa-sn ) } & 42 & 2 \\ 8 & \text { ( hdip oa-ent) } & 25 & 2 \\ 9 & \text { ( oa-sn hdip) } & 28 & 2 \\ 10 & \text { ( oa-sn oa-ent ) } & 35 & 2 \\ 11 & \text { ((c-sn oa-sn ) oa-ent ) } & 27 & 3 \\ 12 & \text { ((cp-ent oa-sn ) hdip ) } & 19 & 3 \\ 13 & \text { ((cp-ent oa-sn ) oa-ent ) } & 30 & 3 \\ & & 554 & \end{array}$

Tree structure
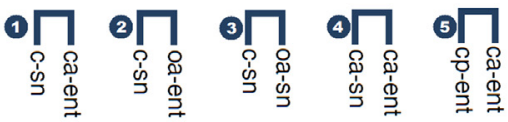

๑П өП

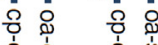

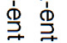
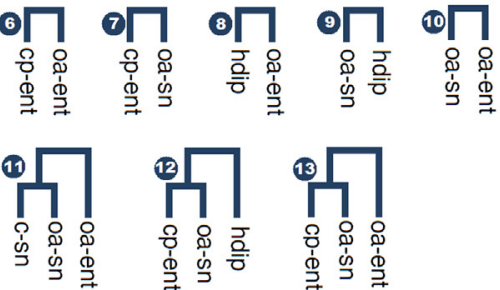

\begin{tabular}{|c|c|c|c|}
\hline TP\# & Terminal string & Occs & Length \\
\hline 1 & (c-sn ca-ent) & 45 & 2 \\
\hline 2 & (c-sn ca-sn) & 38 & 2 \\
\hline 3 & (c-sn oa-sn) & 71 & 2 \\
\hline 4 & (ca-sn ca-ent) & 24 & 2 \\
\hline 5 & $(\mathrm{cp}$-ent c-sn ) & 71 & 2 \\
\hline 6 & (cp-ent ca-ent) & 49 & 2 \\
\hline 7 & ( $c p$-ent $\mathrm{cp}$-sn ) & 23 & 2 \\
\hline 8 & ( cp-ent oa-sn ) & 58 & 2 \\
\hline 9 & ( cp-sn c-sn) & 28 & 2 \\
\hline 10 & ( hdip oa-sn ) & 24 & 2 \\
\hline 11 & ( oa-ent cp-ent ) & 38 & 2 \\
\hline 12 & ( oa-sn hdip) & 32 & 2 \\
\hline 13 & (c-sn ( ca-sn ca-ent)) & 18 & 3 \\
\hline 14 & ( cp-ent ( c-sn ca-ent)) & 40 & 3 \\
\hline 15 & (cp-ent ( c-sn oa-sn )) & 42 & 3 \\
\hline 16 & ( oa-sn ( oa-ent cp-ent)) & 28 & 3 \\
\hline 17 & (( cp-ent c-sn ) ca-ent) & 44 & 3 \\
\hline 18 & (( cp-ent c-sn ) oa-sn ) & 41 & 3 \\
\hline 19 & (( oa-ent cp-ent ) c-sn ) & 29 & 3 \\
\hline 20 & $(($ oa-sn oa-ent $)(\mathrm{cp}$-ent c-sn $))$ & 26 & 4 \\
\hline \multirow[t]{2}{*}{21} & ( oa-sn (( oa-ent cp-ent ) c-sn )) & 23 & 4 \\
\hline & & 792 & \\
\hline
\end{tabular}

DA/Han

ength

2

2

2

2

2

3

3

4

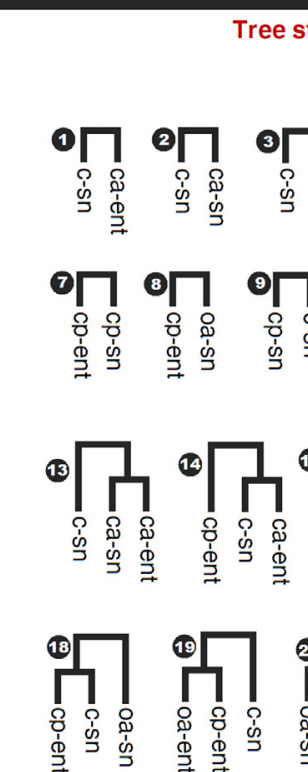

Tree structure

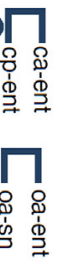

ญิ

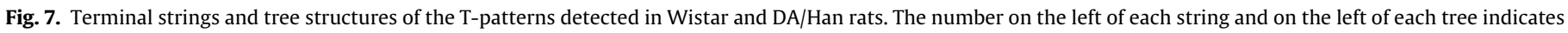

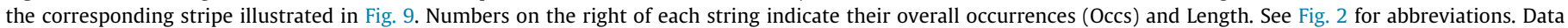
obtained from the analysis of 10 Wistar and 10 DA/Han rats.

DA/Han rats have a behaviour more complex and noticeably more structured in terms of T-patterns. This feature clearly appears even at a first glance by observing the terminal strings and/or the tree structures in Fig. 7, the mean number of detected T-patterns in Fig. 10 or the rasterplots in Fig. 9, the latter presenting the onset of all the T-patterns detected in the two strains. Actually, DA/Han strain performs more different patterns in comparison with Wistar (that is, 21 vs. 13, see Fig. 7). This result demonstrates that the behaviour of the DA/Han, in the $\mathrm{CP}$, is more variable. Such a variability is amplified by the length of detected patterns. Indeed DA/Han rats show longer sequences in comparison with Wistar rats (4length vs. 3-length, see Fig. 7).

These outcomes gain even more emphasis if assessed together with quantitative results (Figs. 4 and 5). Actually, the amount of time spent in walking and sniffing activities by the two strains is very different. Wistar rats do spend much more time in walking activities and noticeably less in sniffing ones (Fig. 5A): with significantly higher frequency, indeed, they do cross the central platform to explore the arms (Fig. 4B); consequently, their permanence in the $\mathrm{CP}$ is significantly lower (Fig. $4 \mathrm{~A}$ ); on the contrary, DA/Han rats

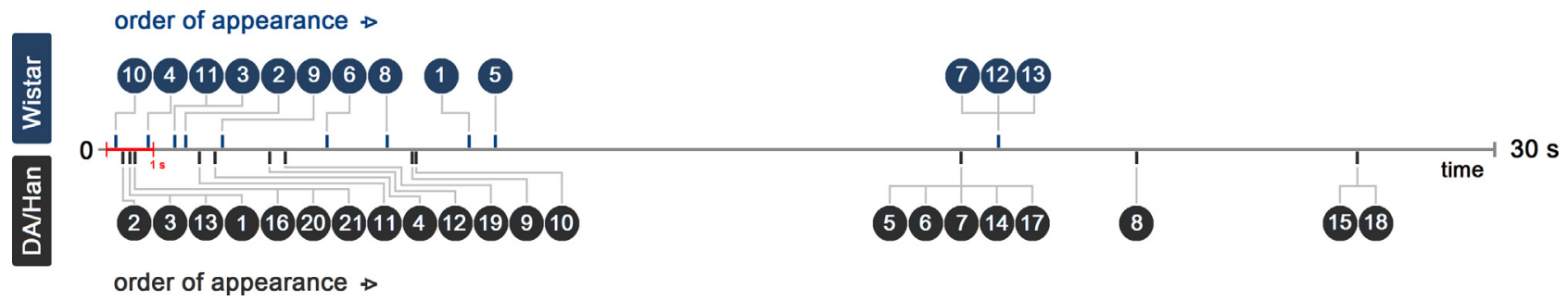

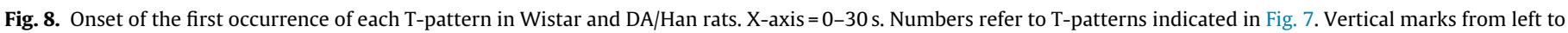
right on the X-axis indicate the onset of the corresponding T-pattern. Data obtained from the analysis of 10 Wistar and $10 \mathrm{DA} / \mathrm{Han}$ rats. 


\section{Wistar}

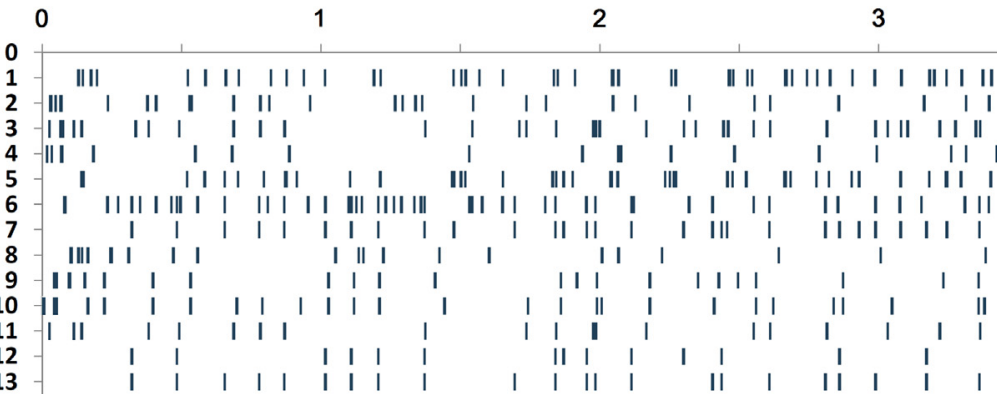

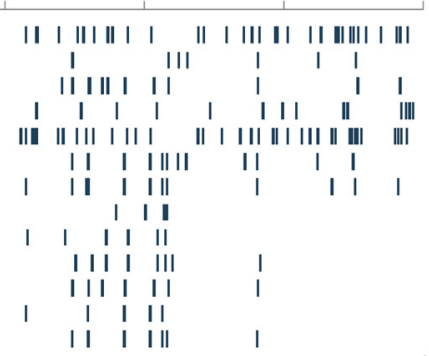

\section{DAHan}

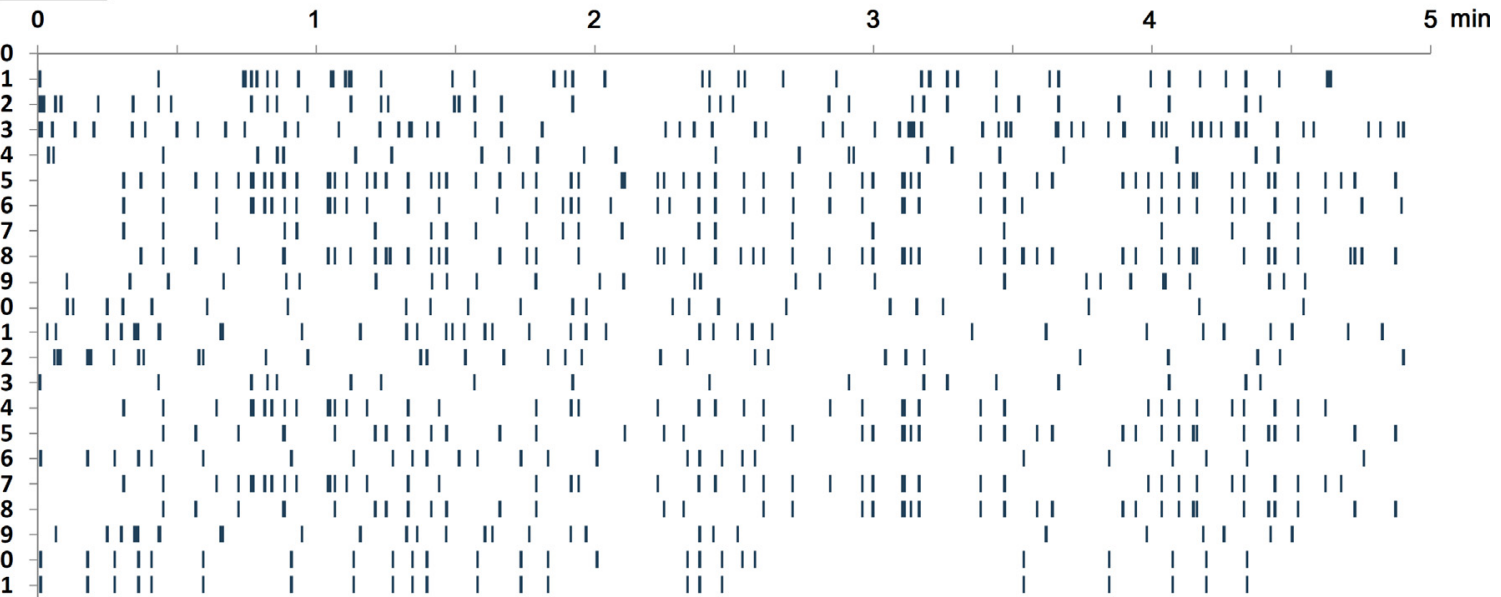

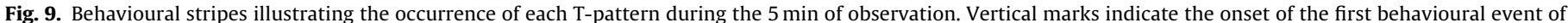

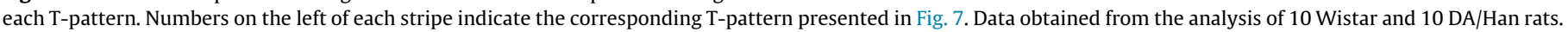

do walk less and sniff much more (Fig. 5A); crossing of CP is significantly reduced both towards the open and the closed arms as well (Fig. 4B); therefore they do present a significantly higher permanence in CP (Fig. 4A) where they, actually, shape the largest extent of their behaviour.

\subsubsection{Two different coping strategies}

Thus, a simple question arises: why should a rat, with a different emotional reactivity, such as the DA/Han, perform more complex and organized patterns of behaviour in the CP of the EPM? The answer probably lies in the different coping strategies of the two strains. The qualitative analysis of all the T-patterns detected, indeed, highlights a quite different organization in terms of performed behavioural sequences. On the one hand several T-patterns

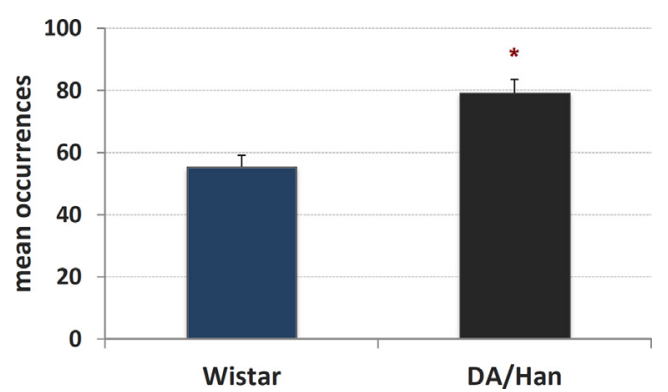

Fig. 10. Mean occurrences $\pm S E$ of T-pattern detected in Wistar and DA/Han rats. $\mathrm{X}$-axis $=$ rodent strain; $\mathrm{Y}$-axis $=$ mean occurrences of T-patterns. ${ }^{*} p<0.05$ difference between the two mean values (Student's $t$ test). Data obtained from the analysis of 10 Wistar and $10 \mathrm{DA} /$ Han rats. in Wistar rats end with the entry in an open or in a closed arm; hence, the Wistar rat, in the novel and potentially dangerous environment, shows a behaviour highly oriented to cross the $\mathrm{CP}$ towards both the open and the closed arm. On the other hand, DA/Han strain shows few patterns concluding with a closed arm entry and never with an open arm entry. On this subject, further details emerge by the evaluation both of latencies, terminal strings and T-patterns' first onset: latencies (Fig. 5) show that Wistar rats, once in the $\mathrm{CP}$, start walking activities significantly earlier than DA/Han; onsets diagram (Fig. 8) illustrates that such an early walking activity is characterized by T-patterns \#10 and \#4, namely, as indicated in Fig. 7, (OA-Sn $\rightarrow$ OA-Ent) and (CA-Sn $\rightarrow$ CA-Ent). Thus, Wistar rats, during the first second (Fig. 8) of EPM exploration, promptly enter both open and closed arms. On the other hand DA/Han rats, with T-patterns \#2, \#3, \#13 and \#1 (Fig. 8), namely, $(\mathrm{C}-\mathrm{Sn} \rightarrow \mathrm{CA}-\mathrm{Sn}),(\mathrm{C}-\mathrm{Sn} \rightarrow \mathrm{OA}-\mathrm{Sn}),(\mathrm{C}-\mathrm{Sn} \rightarrow \mathrm{CA}-\mathrm{Sn} \rightarrow \mathrm{CA}-\mathrm{Ent})$ and $(\mathrm{C}-$ $\mathrm{Sn} \rightarrow$ CA-Ent) (Fig. 7) show, during the first second of interaction with the environment, a behaviour prevalently organized on the basis of sniffing activities and closed arm entries. Such evidences, highly suggestive of a very cautious approach to the environmental exploration, further highlight the emotional reactivity and the higher anxiety level of the DA/Han.

\subsection{Final remarks}

The peculiar characteristics of the central platform, that is, the zone of the elevated plus maze where the animal, on the basis of its emotionality level, selects the arm to explore, calls for an analytical approach able to explore the behaviour in terms of behavioural sequences. In this sense, the multivariate T-pattern analysis is the 
ideal kind of approach. Present research demonstrates, for the first time, that two strains with different emotional reactivity to the environmental stimuli present important differences in terms of the underlying temporal sequences of activities performed in the central platform of the elevated plus maze. These differences have been detailed by using T-pattern analysis, that is, a multivariate approach able to evaluate if sequences of behavioural events are organized on the basis of statistically significant constraints on the interval length separating them. Hence, the utilization of such a multivariate approach to study the behaviour of rats in the central platform can noticeably improve the detection of even subtle features of behaviour, for instance, following the administration of anxiety modulators.

\section{Acknowledgments}

The authors of the present paper are grateful to Vincent Roy and Arnaud Arabo from PSY-NCA, EA4700, Laboratoire de Psychologie et de Neurosciences de la Cognition et de l'Affectivité, Université de Rouen, France, for the helpful comments and suggestions.

\section{References}

Carobrez AP, Bertoglio LJ. Ethological temporal analyses of anxiety-like behavior: the elevated plus-maze model 20 years on. Neurosci Biobehav Rev 2005;29:1193-205.

Casarrubea M, Sorbera F, Crescimanno G. Multivariate data handling in the study of rat behavior: an integrated approach. Behav Res Methods 2009;41:772-81.

Casarrubea M, Sorbera F, Santangelo A, Crescimanno G. Microstructure of rat behavioral response to anxiety in hole-board. Neurosci Lett 2010a:481:82-7.

Casarrubea M, Sorbera F, Santangelo A, Crescimanno G. Microstructural assessment of rodent behavior in the hole-board experimental assay. ACM Digital Library ICPS; 2010b, http://dx.doi.org/10.1145/1931344.1931361, Digital edition.

Casarrubea M, Sorbera F, Santangelo A, Crescimanno G. Learning influence on the behavioral structure of rat response to pain in hot-plate. Behav Brain Res 2011:225:177-83.

Casarrubea M, Sorbera F, Santangelo A, Crescimanno G. The effects of diazepam on the behavioral structure of the rat's response to pain in the hot-plate test: anxiolysis vs pain modulation. Neuropharmacology 2012;63:310-21.

Casarrubea M, Roy V, Sorbera F, Magnusson MS, Santangelo A, Arabo A, et al. Temporal structure of the rat's behavior in elevated plus maze test. Behav Brain Res 2013a;237:290-9.

Casarrubea M, Roy V, Sorbera F, Magnusson MS, Santangelo A, Arabo A, et al. Significant divergences between the temporal structure of the behavior in Wistar and in the spontaneously more anxious DA/Han strain of rats tested in elevated plus maze. Behav Brain Res 2013b;250:166-73.

Casarrubea M, Magnusson MS, Roy V, Arabo A, Sorbera F, Santangelo A, et al. Multivariate temporal pattern analysis applied to the study of rat behavior in the elevated plus maze: methodological and conceptual highlights. J Neurosci Methods 2014;234:116-26, http://dx.doi.org/10.1016/j.jneumeth.2014.06.009.

Casarrubea M, Faulisi F, Sorbera F, Crescimanno G. The effects of different basal levels of anxiety on the behavioural shift analyzed in the central platform of the elevated plus maze. Behav Brain Res 2015a;281:55-61.
Casarrubea M, Jonsson GK, Faulisi F, Sorbera F, Di Giovanni G, Benigno A, et al. T-pattern analysis for the study of temporal structure of animal and human behavior: a comprehensive review. J Neurosci Methods 2015b;239: 34-46.

Chapillon P, Patin V, Roy V, Vincent A, Caston J. Effects of pre and postnatal stimulation on developmental, emotional, and cognitive aspects in rodents: a review. Dev Psychobiol 2002;41:373-87.

Cruz APM, Frei F, Graeff FG. Ethopharmacological analysis of rat behavior on the elevated plus-maze. Pharmacol Biochem Behav 1994:49:171-6.

Espejo EF. Structure of the mouse behaviour on the elevated plus-maze test of anxiety. Behav Brain Res 1997;86:105-12.

Espejo EF, Mir D. Structure of the rat's behaviour in the hot plate test. Behav Brain Res 1993:56:171-6.

Kalueff AV, Tuohimaa P. Grooming analysis algorithm for neurobehavioural stress research. Brain Res Prot 2004;13:151-8.

King SM. Escape-related behaviours in an unstable elevated and exposed environment I. A new behavioural model of extreme anxiety. Behav Brain Res 1999;98:113-26.

Lino-de-Oliveira C, De Lima TC, de Pádua Carobrez A. Structure of the rat behaviour in the forced swimming test. Behav Brain Res 2005;158: 243-50.

Magnusson MS. Discovering hidden time patterns in behavior: T-patterns and their detection. Behav Res Methods Instrum Comput 2000:32:93-110.

Mechan AO, Moran PM, Elliott M, Young AJ, Joseph MH, Green R. A comparison between Dark Agouti and Sprague-Dawley rats in their behaviour on the elevated plus-maze, open-field apparatus and activity meters, and their response to diazepam. Psychopharmacology (Berl) 2002;159:188-95.

Rodgers RJ, Dalvi A. Anxiety, defence and the elevated plus-maze. Neurosci Biobehav Rev 1997;21:801-10.

Rodgers RJ, Johnson NJ. Factor analysis of spatiotemporal and ethological measures in the murine elevated plus-maze test of anxiety. Pharmacol Biochem Behav 1995; 52:297-303.

Rodgers RJ, Lee C, Shepherd JK. Effects of diazepam on behavioural and antinociceptive responses to the elevated plus maze in male mice depend upon treatment regimen and prior maze experience. Psychopharmacology (Berlin) 1992;106:102-10

Roy V, Chapillon P. The positive effects of postnatal handling on defensive burying are more obvious in a situation that enlarge the potential coping responses. Behav Brain Res 2002;136:67-73.

Roy V, Chapillon P. Further evidences that risk assessment and object exploration behaviours are useful to evaluate emotional reactivity in rodents. Behav Brain Res 2004;154:439-48.

Roy V, Lointier L, Chapillon P. The emotional reactivity increase at mid-pregnancy is attenuated in female rats handled during their infancy. Behav Brain Res 2003;145:23-30.

Roy V, Chapillon P, Jeljeli M, Caston J, Belzung C. Free versus forced exposure to an elevated plus-maze: evidence for new behavioral interpretations during test and retest. Psychopharmacology (Berl) 2009;203:131-41.

Spruijt BM, Gispen WH. Behavioral sequences as an easily quantifiable parameter in experimental studies. Physiol Behav 1984;32:707-10.

Treit D, Menard J, Royan C. Anxiogenic stimuli in the elevated plus-maze. Pharmacol Biochem Behav 1993;44:463-9.

Van Den Berg CL, Van Ree JM, Spruijt BM. Sequential analysis of juvenile isolation-induced decreased social behavior in the adult rat. Physiol Behav 1999;67:483-8.

Vanderschuren LJMJ, Spruijt BM, Hol T, Niesink RJM, Van Ree JM. Sequential analysis of social play behavior in juvenile rats: effects of morphine. Behav Brain Res 1996:72:89-95.

Web of Knowledge. According to the search performed in January 2015, using the text string "elevated plus-maze" at the site http://apps.webofknowledge.com. 